Geometry 85 Topology

Volume 5 (2001) 939-945

Erratum 2

Published: 12 December 2001

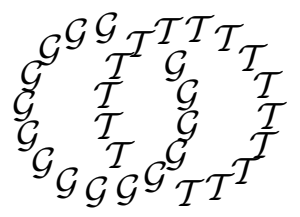

\title{
Lefschetz fibrations on compact Stein surfaces Erratum
}

\author{
Selman Akbulut \\ BURAK OzBAGCI \\ Department of Mathematics \\ Michigan State University \\ MI, 48824, USA \\ Email: akbulut@math.msu.edu, bozbagci@math.msu.edu
}

\begin{abstract}
Sections 3 and 4 of "Lefschetz fibrations on compact Stein surfaces" [Geometry and Topology 5 (2001) 319-334] contained errors due to insufficient care with framing conventions. This note corrects these errors. The following material is a verbatim substitute for half of section 3 and section 4 starting at the heading "General case" on page 330 .
\end{abstract}

\section{General case}

First we represent the 1-handles with dotted-circles stacked over the front projection of the Legendrian tangle. Here we assume that the framed link diagram is in standard form ( $\mathrm{cf}[5])$. Then we modify the handle decomposition by twisting the strands going through each 1-handle negatively once. In the new diagram the Legendrian framing will be the blackboard framing with one lefttwist added for each left cusp. This is illustrated in the second diagram in Figure 10 .

Next we ignore the dots on the dotted-circles for a moment and consider the whole diagram as a link in $S^{3}$. Then we put this link diagram in a square bridge position as in Case 2 (see Figure 10) and find a torus knot $K$ such that all the link components lie on the Seifert surface $F$ of $K$. Now consider the $(P A L F)_{K}$ on $D^{4}$ with regular fiber $F$ as in Proposition 3. We would like to extend $(P A L F)_{K}$ on $D^{4}$ to a PALF on $D^{4}$ union 1-handles. Recall that attaching a 1 -handle to $D^{4}$ (with the dotted-circle notation) is the same as pushing the interior of the obvious disk that is spanned by the dotted circle into the interior of $D^{4}$ and removing a tubular neighborhood of the image from 


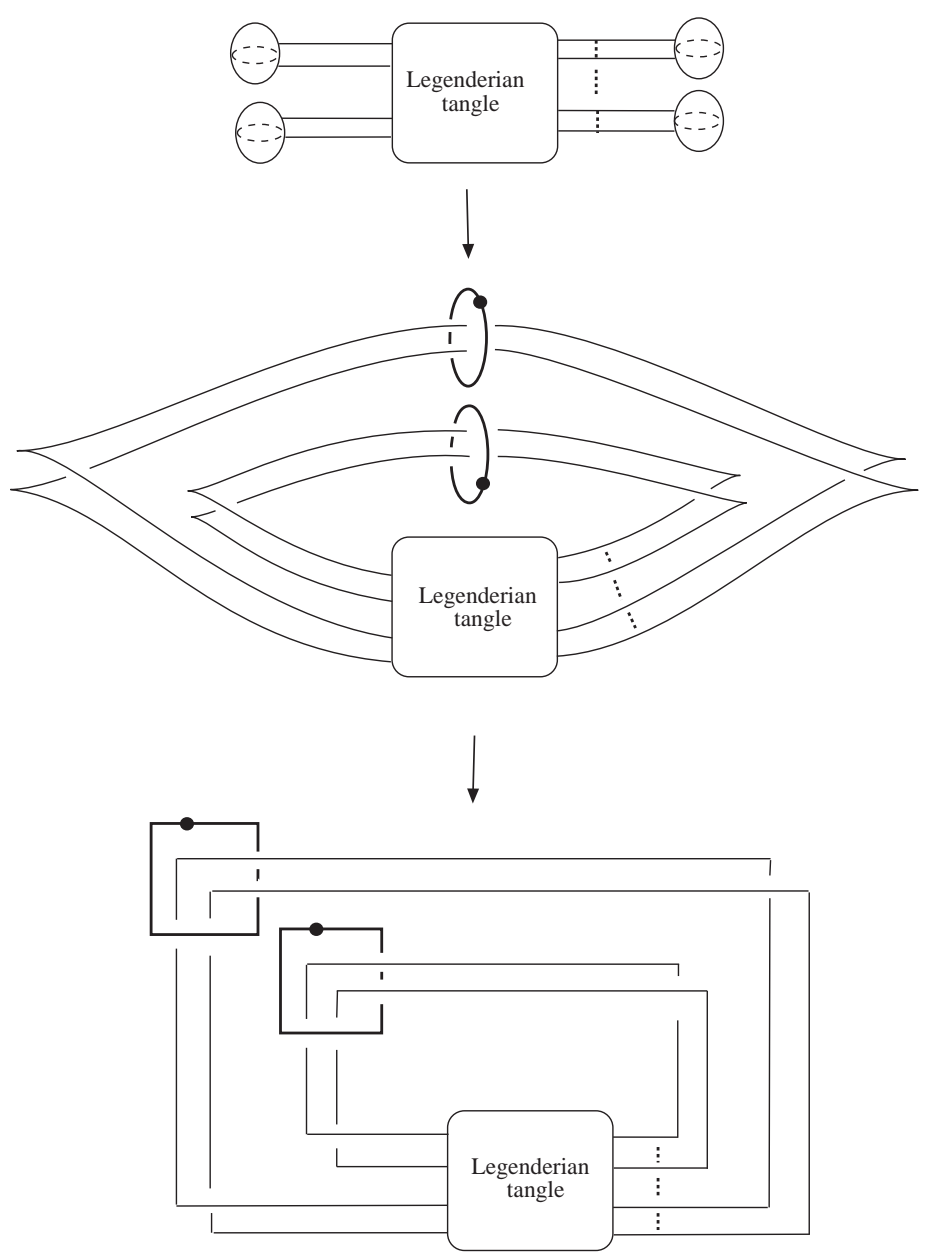

Figure 10: Legendrian link diagram in a square bridge position

$D^{4}$. Before attaching 1-handles we apply the following procedure (cf [11]): We isotope each dotted-circle in the complement of the rest of the link such that it becomes transversal to the fibers of $S^{3} \backslash K$, meeting each fiber only once. (see Figure 11).

Thus by attaching a 1 -handle to $D^{4}$ we actually remove a small 2-disk $D^{2}$ from each fiber of $(P A L F)_{K}$, and hence obtaining a new PALF on $D^{4}$ union a 1-handle. After attaching all the 1-handles to $D^{4}$, we get a new PALF such that the regular fiber is obtained by removing disjoint small disks from $F$. Moreover the attaching circles of the 2-handles are embedded in a fiber in the boundary of the new PALF such that the surface framing of each attaching circle 


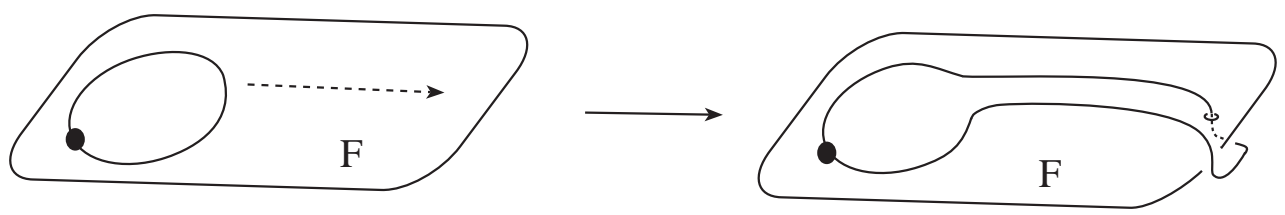

Figure 11: Isotopy of a dotted-circle

is equal to its Legendrian framing. Then Case 2 implies, that is attaching a Legendrian 2-handle at this stage is the same as attaching a Lefschetz 2-handle. Hence, we can extend our PALF on $D^{4} \cup 1$-handles to a PALF on $D^{4} \cup 1$ handles $\cup$ Legendrian 2-handles. The vanishing cycles (hence the monodromy) of the constructed PALF are determined explicitly as follows: We start with the monodromy of the torus knot $K$, extend this over the 1 -handles by identity and then we add more vanishing cycles corresponding to the 2 -handles.

Finally, we note that the $(p, q)$ torus knot in Theorem 2 can be constructed using arbitrarily large $p$ and $q$. Therefore our construction yields infinitely many pairwise nonequivalent PALF's, since for chosen $p$ and $q$ the genus of the regular fiber will be at least $(p-1)(q-1) / 2$.

Conversely, let $X$ be a PALF, then it is obtained by a sequence of steps of attaching 2-handles $X_{0}=D^{2} \times F \leadsto X_{1} \leadsto X_{2} . . \leadsto X_{n}=X$, where each $X_{i-1}$ is a PALF and $X_{i}$ is obtained from $X_{i-1}$ by attaching a 2 -handle to a nonseparating curve $C$ lying on a fiber $F \subset \partial X_{i-1}$. Furthermore this handle is attached to $C$ with the framing $k-1$, where $k$ is the framing induced from the surface $F$. Inductively we assume that $X_{i-1}$ has a Stein structure, with a convex fiber $F \subset \partial X_{i-1}$. By [17] we can start the induction, and assume that the convex surface $F$ is divided by $\partial F$. By the "Legendrian realization principle" of [8] (pp 323-325), after an isotopy of $(F, C), k$ can be taken to be the Thurston-Bennequin framing, and then the result follows by Eliashberg's theorem (L Rudolph has pointed out that, in case of $i=1$ identification of $k$ with Thurston-Bennequin framing also follows from [12]-[15]). Though not necessary, in this process, by using [8] we can also make the framing of $\partial F$ induced from $F$ to be the Thurston-Bennequin framing if we wish.

Remark 4 We show in our proof that the PALF structure on a compact Stein surface $X$ contains a natural smaller PALF $B^{4} \#_{k} S^{1} \times B^{3} \rightarrow D^{2}$ given by the associated torus knot, where $k$ is the number of 1 -handles of $X$.

Remark 5 Our proof shows that by relaxing the condition of positivity, one can identify smooth bounded $4-$ manifolds which are built by 1 - and 2-handles 
with ALF's (allowable Leschetz fibrations over $D^{2}$ 's). In this case in the proof we start with the binding $K \sharp(-K)$ where $K$ is the torus knot, to adjust the framings (ie, we use the general form of [11]). In particular by [17], the boundaries of these manifolds also have contact structures (though not necessarily tight).

\section{Examples}

\subsection{Example 1}

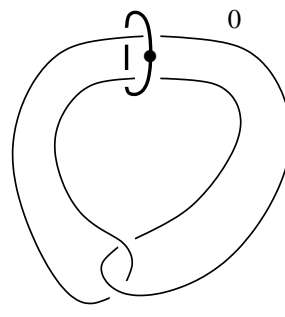

Diagram 1

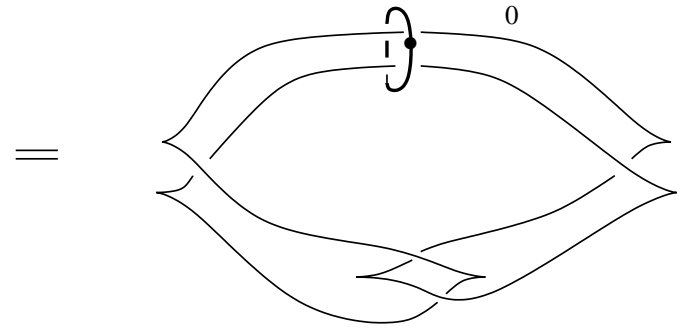

Diagram 2

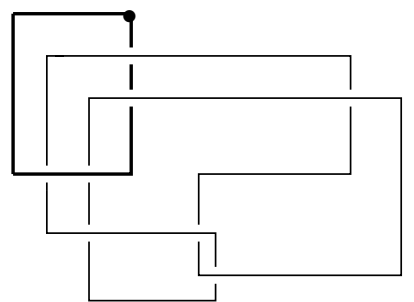

Diagram 3

Figure 12: PALF on a fishtail fiber

In Figure 12, Diagram 1 shows a handle decomposition of a smooth 4-manifold $N$ (a regular neighborhood of a fishtail fiber in an elliptic fibration) which admits a Stein structure. We modify this handle decomposition by twisting the strands going through the 1-handle negatively once, as shown in Diagram 2. In Diagram 3, we put the whole link (including the dotted-circle) in a square bridge position. Note that there are exactly 7 horizontal and 8 vertical lines in the last diagram. Hence according to our algorithm explained above, the Stein surface $N$ admits a PALF with 43 singular fibers where the regular fiber is a genus 21 surface with 2 boundary components. 
Remark 6 The PALF's given by the algorithm of Theorem 5 may not be the most economical ones; sometimes with a little care one can find smaller PALF structures in the sense of having fewer singular fibers. We will illustrate this in the next example.

\subsection{Example 2}

Let $M$ be the Stein surface given as in Figure 13. In the last diagram in Figure 13, we put the feet of the 1-handle onto the binding of the $(2,2)$ torus
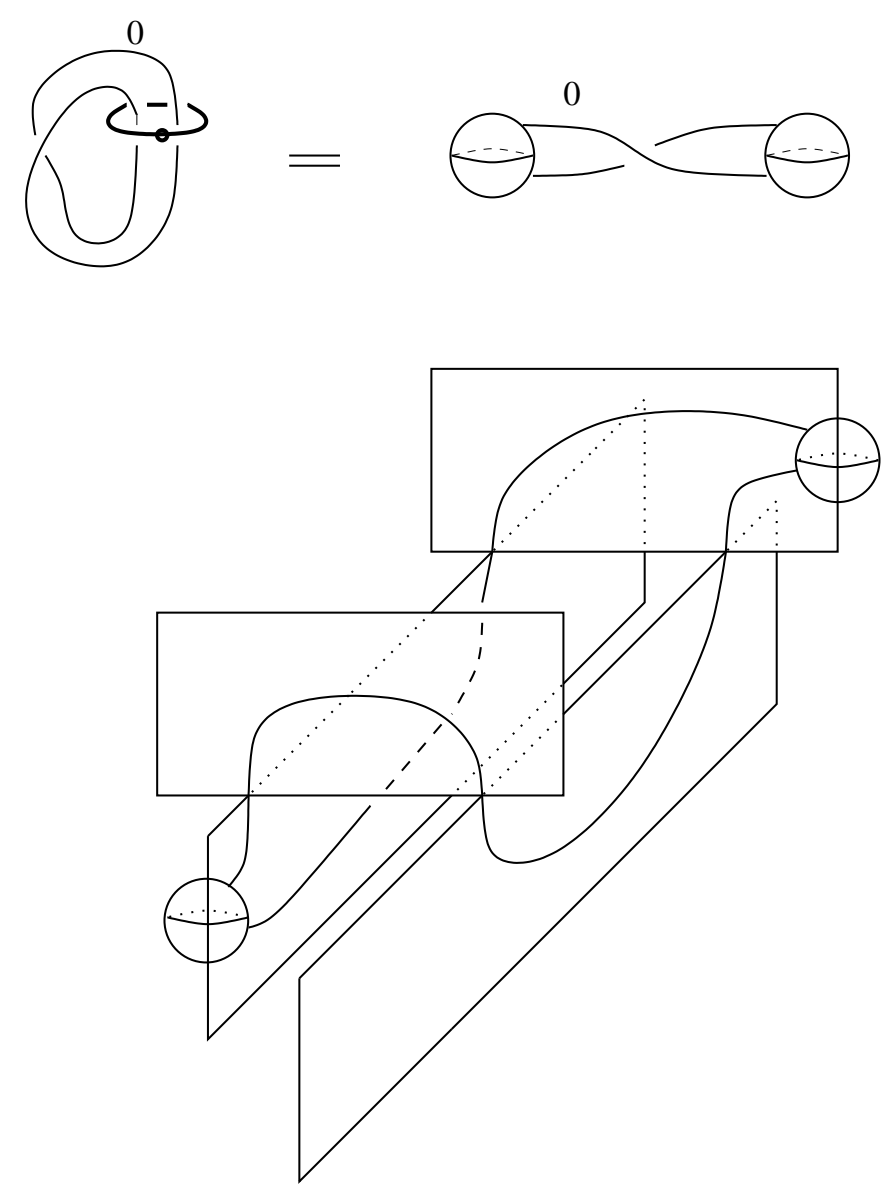

Figure 13

link. Since the attaching region (a pair of 3-balls) of the 1 -handle is in a neighborhood of the binding, we can assume that the pages of the open book 
will intersect the pair of balls transversally as in Figure 14, so that after gluing the 1 -handle to $D^{4}$ we can extend the fibration over the 1 -handle by adding

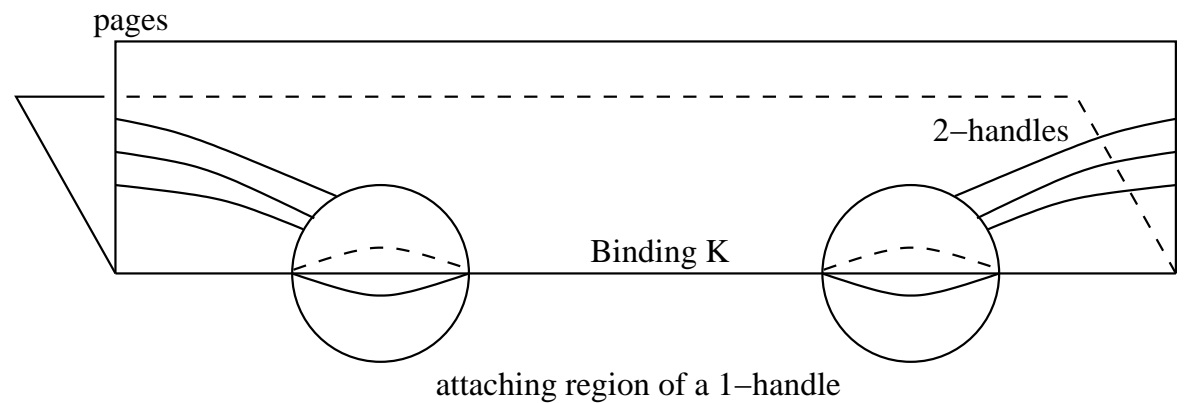

Figure 14: Attaching a 1-handle

a (2-dimensional) 1-handle to the surface of the fibration without altering the monodromy. Note that this is an alternative way of attaching a 1-handle to extend the PALF structure. Hence $M$ admits a PALF with 2 singular fibers where the regular fiber is a punctured torus. The global monodromy of this PALF is the monodromy of the $(2,2)$ torus link, extended by identity over the 1-handle, and composed with a positive Dehn twist corresponding to the 2handle. In Figure 15 we indicate the binding $K$ of the open book decomposition of $\partial M$ obtained from this process.

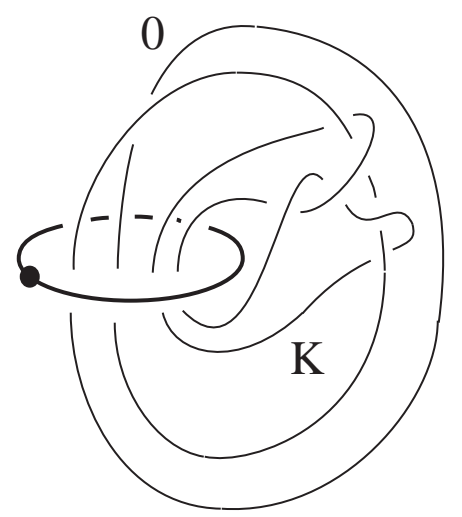

Figure 15

Remark 7 In [1] it was shown that every smooth closed 4-manifold $X$ can be decomposed as a union of two compact Stein surfaces along their boundaries

$$
X=M \cup_{\partial} N .
$$


Hence, every $X$ is a union two PALF's along their boundaries. This gives 4-manifolds a structure somewhat similar to Heegaard decomposition of 3 manifolds (we can consider a 3-dimensional solid handlebody as a Lefschetz fibration over an interval, with fibers consisting of disks). Recall that in [1] there is also a relative version of this theorem; that is, any two smooth closed simply connected h-cobordant manifolds $X_{1}, X_{2}$ can be decomposed as union of Stein surfaces $X_{i}=M \cup_{\varphi_{i}} W_{i}$, where $\varphi_{i}: \partial W_{i} \rightarrow \partial M$ are diffeomorphisms $i=1,2, M$ is simply connected, and $W_{1}, W_{2}$ are contractible manifolds which are diffeomorphic to each other. See also [2] for more about the topology of Stein surfaces.

Bibliography See the bibliography in the original paper:

Selman Akbulut, Burak Ozbagci, Lefschetz fibrations on compact Stein surfaces, Geometry and Topology 5 (2001) 319-334 